\title{
CYLINDRICAL COLLAPSE AND GRAVITATIONAL WAVES
}

\author{
L. Herrera ${ }^{1 * \dagger}$ and N. O. Santos ${ }^{2,3,4 \ddagger}$ \\ ${ }^{1}$ Escuela de Física, Faculdad de Ciencias, \\ Universidad Central de Venezuela, Caracas, Venezuela. \\ ${ }^{2}$ Université Pierre et Marie Curie - CNRS/FRE 2460, \\ LERMA/ERGA, Tour 22-12, 4ème étage, Boîte 142, 4 place Jussieu, \\ 75252 Paris Cedex 05, France. \\ ${ }^{3}$ Laboratório Nacional de Computação Científica, 25651-070 Petrópolis RJ, Brazil. \\ ${ }^{4}$ Centro Brasileiro de Pesquisas Físicas, 22290-180 Rio de Janeiro RJ, Brazil.
}

March 22, 2021

\begin{abstract}
We study the matching conditions for a collapsing anisotropic cylindrical perfect fluid, and we show that its radial pressure is non zero on the surface of the cylinder and proportional to the time dependent part of the field produced by the collapsing fluid. This result resembles the one that arises for the radiation - though non-gravitational - in the spherically symmetric collapsing dissipative fluid, in the diffusion approximation.
\end{abstract}

\footnotetext{
${ }^{*}$ Postal address: Apartado 80793, Caracas 1080A, Venezuela.

$\dagger$ e-mail: laherrera@telcel.net.ve

${ }^{\ddagger}$--mail: nos@cbpf.br and santos@ccr.jussieu.fr
} 


\section{Introduction}

Spherical gravitational collapse of a dissipative fluid produces outgoing radiation which can be modeled with Vaidya spacetime [1]. By using Darmois matching conditions [2], it can be proved that if dissipation within the sphere is described in the diffusion approximation with heat flux, then the pressure on the surface of the collapsing sphere is non zero due to the continuity of the radial flux of momentum [1] (see also [3, 4] and references therein). Indeed, the fact that heat flux does not contribute to the fluid radial pressure within the sphere implies that the pressure does not vanish on the inner part of the boundary surface, because there is radiation pressure on the outer part of that surface. Of course, in the streaming out approximation (i.e. when dissipation within the sphere is described by a radially outgoing null fluid), the fluid radial pressure is continuous across the boundary surface, provided the energy density of such null fluid is continuous too.

It is generally accepted that gravitational waves carry energy, so a source radiating them should lose mass $[5,6,7]$. On the other hand such radiation does not contribute to fluid pressure of the source. Then if one matches a cylindrical non-dissipative fluid to an exterior containing gravitational waves, one may expect that gravitational radiation will exert a non zero pressure on its collapsing surface, like null radiation induces a non-vanishing radial pressure on the boundary surface of a dissipating sphere in the diffusion approximation.

To analyze this problem we studied, by using the Darmois conditions, the matching of a collapsing cylindrical non-dissipative anisotropic fluid to a cylindrically symmetric time dependent exterior in Einstein-Rosen coordinates [8]. Matching conditions for LRS spatially homogeneous collapsing dust space time, have been recently considered in [9].

The paper is organized as follows. In the next section we describe the source and its exterior. In Section 3 the Darmois conditions are presented and in Section 4 the results obtained from its application are exhibited. A specific example is analyzed in Section 5, in order to prove that, in general, the radial pressure does not vanish on the boundary surface of the source. Finally the results are discussed in the last section. 


\section{Collapsing perfect fluid cylinder}

We consider a collapsing cylinder filled with anisotropic non-dissipative fluid bounded by a cylindrical surface $\Sigma$ and with energy momentum tensor given by

$$
T_{\alpha \beta}^{-}=\left(\mu+P_{r}\right) V_{\alpha} V_{\beta}+P_{r} g_{\alpha \beta}+\left(P_{\phi}-P_{r}\right) K_{\alpha} K_{\beta}+\left(P_{z}-P_{r}\right) S_{\alpha} S_{\beta},
$$

where $\mu$ is the energy density, $P_{r}, P_{z}$ and $P_{\phi}$ are the principal stresses and $V_{\alpha}, K_{\alpha}$ and $S_{\alpha}$ are vectors satisfying

$$
V^{\alpha} V_{\alpha}=-1, \quad K^{\alpha} K_{\alpha}=S^{\alpha} S_{\alpha}=1, \quad V^{\alpha} K_{\alpha}=V^{\alpha} S_{\alpha}=K^{\alpha} S_{\alpha}=0 .
$$

We assume the general time dependent cylindrically symmetric metric

$$
d s_{-}^{2}=-A^{2}\left(d t^{2}-d r^{2}\right)+B^{2} d z^{2}+C^{2} d \phi^{2},
$$

where $A, B$ and $C$ are functions of $t$ and $r$. To represent cylindrical symmetry, we impose the following ranges on the coordinates

$$
-\infty \leq t \leq \infty, \quad 0 \leq r, \quad-\infty<z<\infty, \quad 0 \leq \phi \leq 2 \pi
$$

We number the coordinates $x^{0}=t, x^{1}=r, x^{2}=z$ and $x^{3}=\phi$ and we choose the fluid to be comoving in this coordinate system, hence from (2) and (3)

$$
V_{\alpha}=-A \delta_{\alpha}^{0}, \quad K_{\alpha}=C \delta_{\alpha}^{3}, \quad S_{\alpha}=B \delta_{\alpha}^{2} .
$$

The Einstein field equations, $G_{\alpha \beta}=\kappa T_{\alpha \beta}$ for (1), (3) and (5) reduce to five non zero components, but we shall need only the following ones

$$
\begin{array}{r}
G_{11}^{-}=-\frac{B_{, t t}}{B}-\frac{C_{, t t}}{C}+\frac{A_{, t}}{A} \frac{B_{, t}}{B}+\frac{A_{, t}}{A} \frac{C_{, t}}{C}-\frac{B_{, t}}{B} \frac{C_{, t}}{C} \\
+\frac{A_{, r}}{A} \frac{B_{, r}}{B}+\frac{A_{, r}}{A} \frac{C_{, r}}{C}+\frac{B_{, r}}{B} \frac{C_{, r}}{C}=\kappa P_{r} A^{2}, \\
G_{01}^{-}=-\frac{B_{, t r}}{B}+\frac{B_{, t}}{B} \frac{A_{, r}}{A}+\frac{A_{, t}}{A} \frac{B_{, r}}{B}-\frac{C_{, t r}}{C}+\frac{C_{, t}}{C} \frac{A_{, r}}{A}+\frac{A_{, t}}{A} \frac{C_{, r}}{C}=0 .
\end{array}
$$

For the exterior vacuum spacetime of the cylindrical surface $\Sigma$ we take the metric in Einstein-Rosen coordinates [8],

$$
d s_{+}^{2}=-e^{2(\gamma-\psi)}\left(d T^{2}-d R^{2}\right)+e^{2 \psi} d z^{2}+e^{-2 \psi} R^{2} d \phi^{2},
$$


where $\gamma$ and $\psi$ are functions of $T$ and $R$ and for the field equations $R_{\alpha \beta}=0$ we have the gravitational wave field

$$
\psi_{, T T}-\psi_{, R R}-\frac{\psi_{, R}}{R}=0,
$$

and

$$
\gamma_{, T}=2 R \psi_{, T} \psi_{, R}, \quad \gamma_{, R}=R\left(\psi_{, T}^{2}+\psi_{, R}^{2}\right) .
$$

\section{Junction conditions for the collapsing per- fect fluid cylinder}

We take the Darmois junction conditions $[2,10]$, so we suppose that the first fundamental form which $\Sigma$ inherits from the interior metric (3) must be the same as the one it inherits from the exterior metric (8); and similarly, the inherited second fundamental form must be the same. The conditions are necessary and sufficient for a smooth matching without a surface layer.

The equations of $\Sigma$ may be written

$$
\begin{array}{r}
f_{-}=r-r_{\Sigma}=0, \\
f_{+}=R-R_{\Sigma}(T)=0,
\end{array}
$$

where $f_{-}$refers to the spacetime interior of $\Sigma$ and $f_{+}$to the spacetime exterior, and $r_{\Sigma}$ is a constant because $\Sigma$ is a comoving surface forming the boundary of the fluid. To apply the junction conditions we must arrange that $\Sigma$ has the same parametrisation whether it is considered as embedded in $f_{-}$or in $f_{+}$.

Using (11) in (3) we have for the metric on $\Sigma$

$$
d s^{2} \stackrel{\Sigma}{=}-d \tau^{2}+B^{2} d z^{2}+C^{2} d \phi^{2},
$$

where we define the time coordinate $\tau$ only on $\Sigma$ by

$$
d \tau \stackrel{\Sigma}{=} A d t,
$$

and $\stackrel{\Sigma}{=}$ means that both sides of the equation are evaluated on $\Sigma$. We shall take $\xi^{0}=\tau, \xi^{2}=z$ and $\xi^{3}=\phi$ as the parameters on $\Sigma$. 
For the exterior metric (8) using (12) reduces on $\Sigma$ to

$$
d s^{2} \stackrel{\Sigma}{=}-e^{2(\gamma-\psi)}\left[1-\left(\frac{d R}{d T}\right)^{2}\right] d T^{2}+e^{2 \psi} d z^{2}+e^{-2 \psi} R^{2} d \phi^{2},
$$

and is the same as (13) if

$$
\begin{array}{r}
e^{\gamma-\psi}\left[1-\left(\frac{d R}{d T}\right)^{2}\right]^{1 / 2} d T \stackrel{\Sigma}{=} d \tau, \\
e^{\psi} \stackrel{\Sigma}{=} B, \\
e^{-\psi} R \stackrel{\Sigma}{=} C,
\end{array}
$$

where we assume on $\Sigma$

$$
1-\left(\frac{d R}{d T}\right)^{2}>0
$$

so that $T$ is a timelike coordinate.

Equations (14) and (16-18) are the conditions on the interior and exterior metrics imposed by the continuity of the first fundamental form on $\Sigma$.

We turn now to the second fundamental form on $\Sigma$. We need the outward unit normals to $\Sigma$ in $f_{-}$and $f_{+}$; these come from (11) and (12) and are

$$
\begin{array}{r}
n_{\alpha}^{-} \stackrel{\Sigma}{=}(0, A, 0,0), \\
n_{\alpha}^{+} \stackrel{\Sigma}{=} e^{\gamma-\psi}\left[1-\left(\frac{d R}{d T}\right)^{2}\right]_{\underline{\Sigma}}^{-1 / 2}\left(-\frac{d R}{d T}, 1,0,0\right) \\
\stackrel{\Sigma}{=} e^{2(\gamma-\psi)}(-\dot{R}, \dot{T}, 0,0),
\end{array}
$$

where the dot stands for differentiation with respect to $\tau$ introduced by means of (16). Both unit vectors (20) and (21) are spacelike provided (19) is satisfied.

The second fundamental form of $\Sigma$ is

$$
K_{a b} d \xi^{a} d \xi^{b}, \quad a, b=0,2,3,
$$

where $K_{a b}$ is the extrinsic curvature given on the two sides by

$$
K_{a b}^{\mp}=-n_{\alpha}^{\mp}\left(\frac{\partial^{2} x^{\alpha}}{\partial \xi^{a} \partial \xi^{b}}+\Gamma_{\beta \gamma}^{\alpha} \frac{\partial x^{\beta}}{\partial \xi^{a}} \frac{\partial x^{\gamma}}{\partial \xi^{b}}\right) .
$$


The Christoffel symbols are to be calculated from the appropriate exterior or interior metric, (3) or (8), $n_{\alpha}^{\mp}$ are given by (20) and (21), and $x^{\alpha}$ refers to the equation of $\Sigma$ in $f_{-}$or $f_{+}$, namely (11) or (12). The non zero $K_{a b}^{\mp}$ are as follows

$$
\begin{aligned}
& K_{00}^{-} \stackrel{\Sigma}{=}-\frac{A_{, r}}{A^{2}}, \\
& K_{22}^{-} \stackrel{\Sigma}{=} \frac{B B_{, r}}{A}, \\
& K_{33}^{-} \stackrel{\Sigma}{=} \frac{C C_{, r}}{A}, \\
& K_{00}^{+} \stackrel{\Sigma}{=} e^{2(\gamma-\psi)}\{\ddot{T} \dot{R}-\ddot{R} \dot{T} \\
& \left.-\left(\dot{T}^{2}-\dot{R}^{2}\right)\left[\dot{R}\left(\gamma_{, T}-\psi_{, T}\right)+\dot{T}\left(\gamma_{, R}-\psi_{, R}\right)\right]\right\}, \\
& K_{22}^{+} \stackrel{\Sigma}{=} e^{2 \psi}\left(\dot{R} \psi_{, T}+\dot{T} \psi_{, R}\right), \\
& K_{33}^{+} \stackrel{\Sigma}{=}-e^{-2 \psi} R^{2}\left(\dot{R} \psi_{, T}+\dot{T} \psi_{, R}-\frac{\dot{T}}{R}\right) .
\end{aligned}
$$

The complete junction conditions consist of (14) and (16-18) together with the continuity of $K_{a b}$ across $\Sigma$.

\section{Results}

In this section, we use the interior and exterior field equations to write the boundary conditions in a concise form. In order to do that we first derive some useful formulae.

From (16) we have that

$$
e^{2(\gamma-\psi)}\left(\dot{T}^{2}-\dot{R}^{2}\right) \stackrel{\Sigma}{=} 1,
$$

and from (17) and (18)

$$
R \stackrel{\Sigma}{=} B C .
$$

Using (14) we can differentiate (31) yielding

$$
\dot{R} \stackrel{\Sigma}{=} \frac{(B C)_{, t}}{A},
$$


and from the continuity of $K_{22}$ and $K_{33}$ with (17) and (18) we obtain

$$
\dot{T} \stackrel{\Sigma}{=} \frac{(B C)_{, r}}{A} .
$$

Now differentiating (32) and (33) with (6), (7) and (14) we can write the relation

$$
\begin{array}{r}
\ddot{T} \dot{R}-\ddot{R} \dot{T} \stackrel{\Sigma}{=} \frac{1}{A^{4}}\left\{(B C)_{, t}\left[B_{, t}(A C)_{, r}+C_{, t}(A B)_{, r}\right]\right. \\
\left.+(B C)_{, r}\left[+\kappa P_{r} A^{3} B C-A B_{, t} C_{, t}-A_{, r}(B C)_{, r}-A B_{, r} C_{, r}\right]\right\} .
\end{array}
$$

From the continuity of $K_{00}$ and $K_{22}$ and with (10), (17), (30), (31), (33) and (34) we get the expression

$$
\frac{1}{A^{4}}\left[\left(B_{, t} C_{, r}-B_{, r} C_{, t}\right)^{2}+\kappa P_{r} A^{2}(B C)_{, r}^{2}\right] \stackrel{\Sigma}{=}\left(\dot{T}^{2}-\dot{R}^{2}\right)^{2} \psi_{, T}^{2} .
$$

Differentiating (17) and (18) with respect to (14) and (16) we obtain

$$
\begin{array}{r}
e^{2 \psi-\gamma}\left[1-\left(\frac{\dot{R}}{\dot{T}}\right)^{2}\right]^{-1 / 2} \psi_{, T} \stackrel{\Sigma}{=} \frac{B_{, t}}{A}, \\
e^{-\gamma}\left[1-\left(\frac{\dot{R}}{\dot{T}}\right)^{2}\right]^{-1 / 2}\left(\frac{\dot{R}}{\dot{T}}-R \psi_{, T}\right) \stackrel{\Sigma}{=} \frac{C_{, t}}{A} .
\end{array}
$$

Then from the continuity of $K_{22}$ and $K_{33}$ with (36) and (37) we have

$$
\frac{1}{A^{2}}\left(B_{, t} C_{, r}-B_{, r} C_{, t}\right) \stackrel{\Sigma}{=}\left(\dot{T}^{2}-\dot{R}^{2}\right) \psi_{, T}-\dot{T} \dot{R} \psi_{, R}
$$

Finally substituting (38) into (35) and considering (10) and (33) we obtain

$$
\kappa P_{r} \stackrel{\Sigma}{=} e^{2(\psi-\gamma)} \psi_{, R}^{2}\left(2 \frac{\psi_{, T}}{\psi_{, R}} v-\frac{v^{2}}{1-v^{2}}\right),
$$

where $v \stackrel{\Sigma}{=} d R / d T$ denotes the radial velocity of the boundary surface. The result (39)shows that the radial pressure $P_{r}$ on the surface $\Sigma$ of the collapsing perfect fluid is non zero. The reason for this might be due to the flux of 
momentum of the gravitational wave emerging from the cylinder. If the cylindrical fluid source is static then $v \stackrel{\Sigma}{=} 0$ and $P_{r} \stackrel{\Sigma}{=} 0$ as expected.

However, it might be argued that $P_{r} \stackrel{\Sigma}{=} 0$ is always satisfied in cylindrical collapse, leading to an equation for $v$, which reads

$$
v \stackrel{\Sigma}{=} \frac{1}{x}\left[-1 \pm\left(1+x^{2}\right)^{1 / 2}\right],
$$

with $x=4 \psi_{, T} / \psi_{, R}$. By means of an example we shall prove in the next section that this is not the case.

\section{A pulse solution}

Let us now consider a cylindrical source which is static for a period of time until it starts contracting and emits a sharp pulse of radiation travelling outward from the axis. Then, the function $\psi$ can be written as [11]

$$
\psi=\frac{1}{2 \pi} \int_{-\infty}^{T-R} \frac{f\left(T^{\prime}\right) d T^{\prime}}{\left[\left(T-T^{\prime}\right)^{2}-R^{2}\right]^{1 / 2}}+\psi_{s t},
$$

In $(41) \psi_{s t}$ represents the Levi-Civita static solution [12], and $f(T)$ is a function of time representing the strength of the source of the wave and it is assumed to be of the form

$$
f(T)=f_{0} \delta(T),
$$

where $f_{0}$ is a constant and $\delta(T)$ is the Dirac delta function. It can be shown that (41) satisfies the wave equation (9). Then we get

$$
\begin{array}{r}
\psi=\psi_{s t}, \quad T<R ; \\
\psi=\frac{f_{0}}{2 \pi\left(T^{2}-R^{2}\right)^{1 / 2}}+\psi_{s t}, \quad T>R .
\end{array}
$$

The function $\psi$, as well as its derivatives, is regular everywhere except at the wave front determined by the surface $T=R$, followed by a tail decreasing with $T$. Using $\psi_{s t}=\alpha-\beta \ln R$, with $\alpha$ and $\beta$ being constants, and (44) we obtain

$$
x \stackrel{\Sigma}{=} \frac{4 f_{0} T R}{2 \pi \beta\left(T^{2}-R^{2}\right)^{3 / 2}-f_{0} R^{2}} .
$$


From (45) we see that as $T$ increases, the system tends asymptotically to a static situation, and for a sufficiently large values of $T$ we have

$$
x \stackrel{\Sigma}{\approx} \frac{2 f_{0} R}{\pi \beta T^{2}} .
$$

which is a positive quantity. On the other hand for values of $T$ larger than, but sufficiently close to $R$, we have that $x$ is negative. This implies that for some value of $T$ (say $T_{0}$ ) the denominator in (45) vanishes and $x$ tends to $\mp \infty$ as $T$ goes from $T_{0}-0$ to $T_{0}+0$. This in turn implies, because of (40) (where only the upper sign before the square root in (40) has to be considered because $\left.v^{2}<1\right)$, that in the infinitesimal time interval $\left(T_{0}-0, T_{0}+0\right)$ the velocity $v$ changes from -1 to +1 . This of course is impossible. Therefore, condition (40) cannot be satisfied in this example and $P_{r} \neq 0$ on $\Sigma$.

It is worth noticing that for sufficiently large values of $T, \Psi_{,_{R}} \Psi_{,_{T}}>0$ on $\Sigma$. This quantity, which is related to the rate of change of the $C$ energy, is shown to be negative in [7]. However that proof is valid only for large values of $R$, which of course is not the case here.

It is also worth noticing that, in general, the static exterior spacetime, i. e. Levi-Civita spacetime, cannot be matched to a collapsing cylinder with source (1). Indeed, considering that the exterior spacetime is static, $\psi(R)$, then we have from (17), (33) and the continuity of $K_{22}$ that $B \stackrel{\Sigma}{=} B(r)$ and

$C \stackrel{\Sigma}{=} C(r)$, which in turn implies because of $(31)$ that $R \stackrel{\Sigma}{=}$ constant showing that the source cannot be collapsing. This result has been shown before for the particular case of a collapsing dust cylinder [13].

\section{Conclusion}

The main result of this work is that the radial pressure on the surface of a collapsing anisotropic perfect fluid cylinder is non zero (39). If the system is static we reobtain the usual result that the radial pressure is zero on the boundary surface (the same happens in the slowly evolving case considered by Bondi [14]).

The physical interpretation of the non-vanishing surface pressure might be justified through the continuity of the radial flux of momentum across the boundary surface. However, based on this interpretation what follows is that the collapsing dust cylinder $\left(P_{r}=0\right)$ should not radiate gravitational 
waves, the exterior spacetime then belongs to the class of time dependent systems without news, as discussed by Bondi et al in [6]. Parenthetically Bondi suggests that pressure-free dust would be the most clear cut example of such non-radiating time dependent systems.

Finally, we can state that if a cylinder with source, as described in (1), is collapsing, it will always produce gravitational waves outside the source, implying the presence of non-vanishing radial pressure on the boundary of the source.

\section{References}

[1] Santos N O 1985 Mon. Not. R. Astron. Soc. 216403

[2] Darmois G 1927 Mémorial des Sciences Mathématiques, GauthierVillars, Paris, Fasc. 25

[3] Bonnor W B, de Oliveira A K G and Santos N O 1989 Phys. Rep. 181 269

[4] Herrera L and Santos N O 1997 Phys. Rep. 28653.

[5] Marder L 1958 Proc. Roy. Soc. Lond. A244 524

[6] Bondi H, van der Burg M G J and Metzner A W K 1962 Proc. Roy. Soc. Lond. A269 21

[7] Apostolatos T A and Thorne K S 1992 Phys. Rev. D 462435

[8] Einstein A and Rosen N 1937 J. Franklin Inst. 22343

[9] Tod P anf Mena F 2004 Phys. Rev. D 70104028

[10] Bonnor W B and Vickers P A 1981 Gen. Rel. Grav. 1329

[11] Carmeli M 1982 Classical Fields: General Relativity and Gauge Theory, John Wiley \& Sons, New York

[12] Levi-Civita T 1919 Rend. Acc. Lincei 28101 
[13] Gutti S, Singh T P, Sundararajan P A and Vaz C 2002 arXiv:grqc/0212089

[14] Bondi H 1990 Proc. Roy. Soc. Lond. A427 259 